\title{
Research of the Technology and Application of Spacecraft Assembly Data-package Construction Based on MBD
}

\author{
Bi Le Wan ${ }^{1}$, Chang Yu Chen ${ }^{1}$ and Wen Xing He ${ }^{1, a}$ \\ ${ }^{1} 1$ Beijing Institute of Spacecraft Environment Engineering, Beijing 100094, China
}

\begin{abstract}
In order to implement spacecraft assembly based on MBD, this article proposes a method to construct datapackage automatically based on 3D model. First, an Assembly Digital Mock-up (ADMU) is constructed. Second, we propose the way of assembly process design based on 3D structural process design. Third, we construct the product data-package automatically based on the relationship among ADMU, the process files and production parameters Finally, the paper describes the application of the technology, and analyses the effect of the scheme during a satellite development. The technology and systems should be applied on other sophisticated products development, with some essential modifications.
\end{abstract}

\section{Introduction}

For international aerospace companies, Product Datapackage is an assemblage of product data, specification and form, and its management is also an important work for product quality. For example, the ECSS (Europe Cooperation for Space Standardization) requires that the data-package must include manufacture data, assembly data, test data and event record, and the data-package is the foundation of the product delivery. Meanwhile, other companies or organizations propose similar requirements [1].

Traditionally, with two-dimensional drawings and files, people design in Computer Aided Process Planning (CAPP), and all product data need to deliver manually among departments, specialties, and careers, especially between the design department and the assembly department. So traditionally the data-package is constructed manually, but the development of Model Based Definition technology makes it possible to construct the data-package automatically [2].

Researchers have made much research on about Model Data Definition [3-4], Digital Mock-up construction [2] [5-6], Process Planning and Alteration Control [7-11]. But those papers didn't describe the technology of Data-package Construction Based 3D model and related systems. Scheme of $3 \mathrm{~d}$ structural process design shown in Fig. 1.

In order to fulfill the requirement of the spacecraft assembly, the paper proposes 3D Data-package Construction scheme based on Assembly Process Digital Mock-up (APDM) and 3D Structural Process Design. The paper defines a special parameter named as Structural Assembly Parameter, and proposes the 3D Data-package Construction scheme based on it. The paper describes the workflow and introduces the application of a satellite development.

\section{The Status of Spacecraft Digital Assembly}

Currently, the spacecraft digital assembly has already realized structural process design based on threedimension. The general idea is using process digital mock-up which inherits and refactors design digital mock-up as the only data source. Firstly, we conduct the design and simulation of process planning. After that, we conduct process explicit design and edition after the process of simulation verification. Materiels such as manufacturing resources, manufacturing information and design information can be acquired directly from process digital mock-up. Then the relevant process files, including media such as models, videos and pictures, are delivered to MES as production basis after approval. After that, the staff responsible for dispatch, quality or production conduct work and record production parameters through MES system. After executing that, relevant production data will be uploaded to process design system as data resources of assembly datapackage.

\section{The Definition and Construction of Spacecraft Assembly data-package}

Before you begin to format your paper, first write and save the content as a separate text file. Keep your text and graphic files separate until after the text has been formatted and styled. Do not use hard tabs, and limit use of hard returns to only one return at the end of a paragraph. Do not add any kind of pagination anywhere

\footnotetext{
a Corresponding author: hewenxing@163.com
} 
in the paper. Do not number text heads-the template will do that for you.

Finally, complete content and organizational editing before formatting. Please take note of the following items when proofreading spelling and grammar.

\subsection{The definition of spacecraft assembly data- package}

According to 'The quality and reliability data package space product and its application' and 'Research on the basic theory of product data-packages and product maturity', China's space industry defines data package as the product quality and reliability data-packages. It was born from the process of whole satellites and parts in the aerospace products from the design to the delivery, and it mainly record the product quality and reliability of the relevant documents and process information. The space product data packages are reflected in the whole process of the design, manufacture, testing and delivery of the product data and integrated into results of quantitative control of all technical activities. At the same time, product data-package is the object record of product realization process and the realization result.

In summary, spacecraft assembly data-package type faces to spacecraft system design, assembly and test, test and launchment and assembly of the entire process, including the overall design data, process design data, production data, test data and objective record data.

\subsection{Constituent elements of spacecraft assembly data-package}

With the development of 3D, the 3D data-packages of spacecraft can be divided into design data, process design data, production data, test data and objective data. The design data generally includes three-dimensional design data (such as three-dimensional model, structured data), technical documents, etc., as well as the relevant alteration of design data, technical notice and technical problem bills. Process design data including process planning (including formal process files and temporary process files), the implementation of change records, simulation video, workflow, process route, drawings and process alternation etc. Production data including production status data, executed production parameters, material data and the person who implemented. The inspection data includes the actual measurement parameters, the executor and the re-inspection information. The objective record data mainly includes the data of the objective state of the product, such as photos, video and so on.

All kinds of data in the three-dimensional assembly data exist in the form of electronic data, or a small amount of paper data (such as product transfer, review records, etc.). But comparing with the traditional assembly data-packages, the electronic data of threedimensional assembly data-packages has the character of bidirectional correlation, such as the relation among counting machine and process digital mock-up, process digital mock-up and process rules, process rules and production data, production data and the implementation process, which makes the application of threedimensional assembly data-packages convenient and proficient.

\section{The Construction Scheme of Three- dimensional Assembly Data-packages}

On the basis of constructing the digital mock-up and the 3D structural process design, the basic frame of the Built BOM is using Process BOM constructing the frame of Built BOM (known as three-dimensional assembly data-package). After that, it gradually builds a Built BOM with the implement. Then the Executed Process File BOM is constructed by combining the Process File BOM with the production data, and we can build a complete Built BOM through the real process tree and the bidirectional association of Process BOM. The general plan is shown as below. Assembly data-package construction shown in Fig. 1.

\subsection{Definition of assembly structural parameter (ASP)}

In the process of constructing 3D assembly datapackage, the ASP is key to achieve this object. According to figure 1 and figure 2 , the ASP is defined in the process file, which include three elements, the type of keywords, the main object product or related product, technical requirement and executed parameters.

Type keywords is the basis to determine the execution record type, including "install, remove and record", such as "XX product has been installed in place", "XX product has been removed" and "the insulation resistance of XX product is --". The type of installation and remove are critical to generate Built BOM, while the record type is the supplement of these two types. The keyword is determined when the system uses the definition of a record template.

Main object or relational object is the keyword to judge the ASP, and make it associated with the Process BOM, which determines the state or parameter of Built BOM. The keywords are determined by the process designer during process design.

Technical requirements are keywords of process requirements, and it is used to determine the type of production parameters. The keywords are determined by the process designer in the process design.

The production parameters are used to record the total actual production data, which are filled by inspectors, and automatically feedback to Built BOM after finishing the corresponding completion.

\subsection{Assembly data-packages construction process based on ASP}

The workflow as shown: 


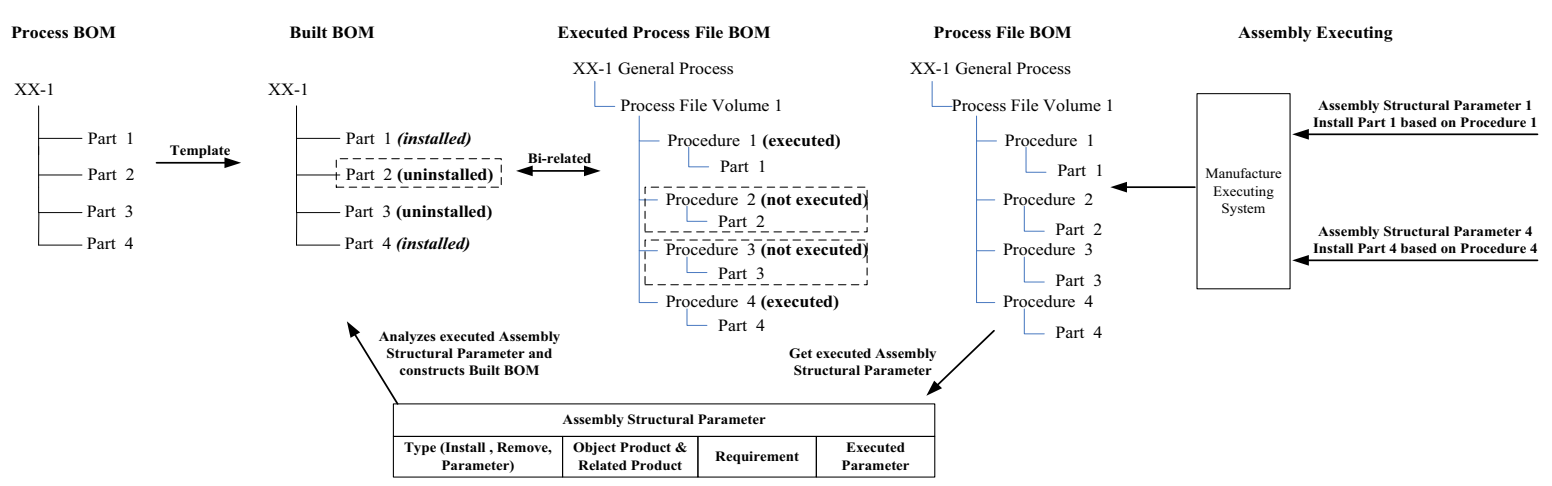

Fig.1 Assembly Data-package Construction

- Operation or inspection personnel in the MES check the three-dimensional process files and implement the assembly operation based on the process requirements. After that, they sign in the execution record of the threedimensional process files and the implementation of the process in the MES system.

- Pending the completion of the process or procedure (i.e. completed deployment), MES will return process or step file that contain production data and ASP to the threedimensional assembly data package system. In addition, photos and other objective data are returned by the inspection personnel through the MES tools after associating the photograph with process.

- Analysis the returned ASP (record of implementation of execution) and determine its type. Specifically, it is

\subsection{The Frame of 3D Assembly Data-packages System}

3D assembly data-packages system contains Interface layer, Data flow, and Application layer, and the overall framework is shown in the following figure. The acquisition of the data is collected through the integration of MES and Webservice interface, and production data analysis is achieved by collecting the XML file returned through integration of the interface. Framework of 3D assembly data-package system shown in Fig. 2 .

\section{Interface}

Data Flow

\section{Application}

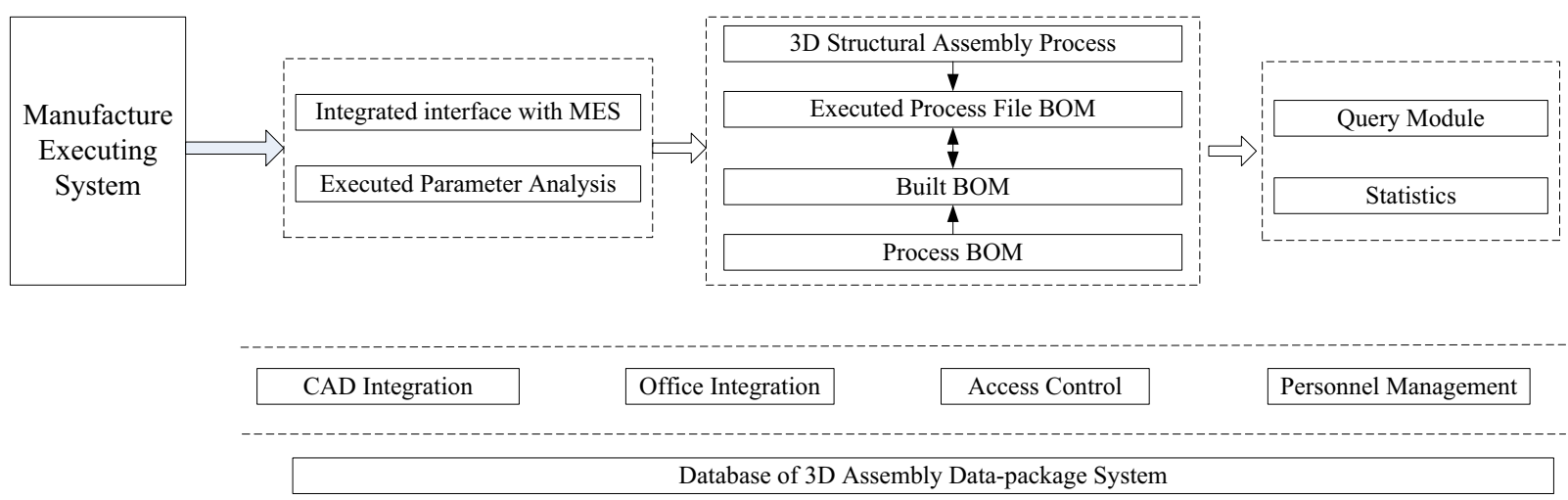

Fig. 2 Framework of 3D Assembly Data-package System

judged whether it belongs to the installation, removal or record.

- If it belongs to installation, first constructs Executed Process File BOM according to Process File BOM and analyzes main object of ASp. After that, connect the corresponding product to Built BOM. Besides, connects ASP with its parameters, and connects objective record data connected with implementation process files, such as photos, with Built BOM.

- If it belongs to demolition, removes corresponding product from Built $\mathrm{BOM}$ after generating the main $\mathrm{BOM}$ and analyzes main object. Then connects ASP.

- If it belongs to record, record ASP and its parameters to the corresponding product of Built BOM after generating the main tree and analyzes main object.

\section{Application}

In the process of $3 \mathrm{D}$ assembly, automatic creation of $3 \mathrm{D}$ assembly data is realized on a certain type of product based on the construction of the digital prototype and the completion of the three-dimensional structure of the process design:

- Complete the association of design digital prototype, process digital prototype, process documents and production implementation of the relationship, and realize the traceable query of association, as shown in the following figure 
- Realize the construction of digital experimental prototype shown in the form of tree structure, in which products have been installed tare represented in white, the uninstalled or dismantled products is shown in red .Besides, implementation of product model and types of real are consistent.

- Realize the association query of product object, implementation process and objective record of data and personalized comprehensive query and data statistics based on template.

\section{Conclusion}

This paper introduces the construction and application of spacecraft assembly data based on MBD. On the base of construction of the digital prototype of the model and the $3 \mathrm{D}$ structure design, this paper propose the construction scheme and process of 3D assembly data, and illustrate the application effect by the case of model application.

\section{Acknowledgement}

This research is supported by Natural Science Foundation of China (51405025).

\section{References}

1. Hongtao Liu. Research on commercialization management evaluation in aerospace enterprise [D]. Hebei University of Technology. 2012.

2. Wenxing He, Bile Wan, Bin Zhang, Xinghui Wu. Research of the technology and application of process digital mock-up for spacecraft assembly. Advanced Materials Research. 2014(1037):447-452.

3. Yuqing Fan. Model Based Definition Technology and Its Practices. Aeronautical Manufacturing Technology. 2012(06):42-47.

4. Quintana V, Rivest L, Prellerin R, et al. Will ModelBased Definition replace engineering drawings throughout the product lifecycle? A global perspective from aerospace industry $[\mathrm{J}]$. Computers in Industry. 2010, 61(5):497-508.

5. Zorriassatine F, Wykes C, Parkin R, et al. A survey of virtual prototyping techniques for mechanical product development $[\mathrm{J}]$. Proceedings of the Institution of Mechanical Engineers, Part B (Journal of Engingeering Manufacture). 2003, 217(B4):513530 .

6. sebastien Gagne, Clement Fortin. Application of the CMII Model to an integrated engineering and manufacturing development environment. Ingt $\mathrm{J}$ Ineract Des Manuf. 2007(1):5-13.

7. Alemanni M, Destefanis F, Vezzetti E. Model-based definition design in the product lifecycle management scenario [J]. International Journal of Advanced Manufacturing Technology. 2011,52(14):1-14
8. Lihong Qiao, Jin Zhang. Some Key Issues in ThreeDimensional Digital Process Planning and Their Research. Aerospace Manufacturing Technology. 2010(1):29-32.

9. Tao Yang, Zhongyi Mei. Aircraft Full ThreeDimensional Implementation Technology Based on Mbd. Aerospace Manufacturing Technology. 2013(08):26-31.

10. Yuqing Fan, Yan Liang, Guocheng Feng. Data Organization of MBD-Based Assembly and Its Implementation, Aviation Maintenance \& Engineering .6(2010)56-59.

11. In-Ho Song, Sung-Chong Chung. Synthesis of the digital mock-up system for heterogeneous CAD assembly. Computers in Industry. 2009(60):285-295.

12. Jiajun Yuan. Study of Aerospace Product Maturity. Spacecraft Engineering. 2011(20):1-7. 